\title{
Effect of Space Flight on the Behavior of Human Retinal Pigment Epithelial ARPE-19 Cells and Evaluation of Coenzyme Q10 Treatment
}

\section{Francesca Cialdai}

ASAcampus Joint Laboratory, ASA Res. Div., Department of Experimental and Clinical Biomedical

Sciences "Mario Serio", Università degli Studi di Firenze

\section{Davide Bolognini}

University of Florence Department of Experimental and Clinical Medicine: Universita degli Studi di

Firenze Dipartimento di Medicina Sperimentale e Clinica

\section{Leonardo Vignali}

ASAcampus Joint Laboratory

\section{Nicola lannotti}

Department of Life Sciences, Università degli Studi di Siena

\section{Stefano Cacchione}

University of Rome La Sapienza Department of Biology and Biotechnology Charles Darwin: Universita degli Studi di Roma La Sapienza Dipartimento di Biologia e Biotecnologie Charles Darwin

\section{Alberto Magi}

Department of Information Engineering, Università degli Studi di Firenze

\section{Michele Balsamo}

Kayser Italia srl

\section{Gianluca Neri}

Kayser Italia srl

\section{Alessandro Donati}

Kayser Italia srl

\section{Marco Vukich}

Kayser Italia srl

\section{Monica Monici}

ASAcampus Joint Laboratory

\section{Sergio Capaccioli}

University of Florence Department of Experimental and Clinical Biomedical Sciences Mario Serio:

Universita degli Studi di Firenze Dipartimento di Scienze Biomediche Sperimentali e Cliniche Mario Serio Matteo Lulli ( $\triangle$ matteo.lulli@unifi.it)

Università degli Studi di Firenze https://orcid.org/0000-0002-8528-4094 


\section{Research Article}

Keywords: retina, microgravity, radiations, retinopathy, space flight.

Posted Date: July 14th, 2021

DOl: https://doi.org/10.21203/rs.3.rs-675406/v1

License: (c) (i) This work is licensed under a Creative Commons Attribution 4.0 International License. Read Full License

Version of Record: A version of this preprint was published at Cellular and Molecular Life Sciences on October 29th, 2021. See the published version at https://doi.org/10.1007/s00018-021-03989-2. 
Effect of space flight on the behavior of human retinal pigment epithelial ARPE-19 cells and evaluation of coenzyme Q10 treatment

Francesca Cialdai $^{1}$, Davide Bolognini ${ }^{2}$, Leonardo Vignali ${ }^{1}$, Nicola lannotti ${ }^{3}$, Stefano Cacchione $^{4}$, Alberto Magi ${ }^{5}$, Michele Balsamo ${ }^{6}$, Marco Vukich ${ }^{6}$, Gianluca Neri ${ }^{6}$, Alessandro Donati ${ }^{6}$, Monica Monici ${ }^{1}$, Sergio Capaccioli ${ }^{7}$, and Matteo Lulli ${ }^{7},{ }^{*}{ }^{*}$

${ }^{1}$ ASAcampus Joint Laboratory, ASA Res. Div., Department of Experimental and Clinical Biomedical Sciences "Mario Serio", Università degli Studi di Firenze, Italy; ${ }^{2}$ Department of Experimental and Clinical Medicine, Università degli Studi di Firenze, Italy; ${ }^{3}$ Department of Life Sciences, Università degli Studi di Siena, Italy; ${ }^{4}$ Department of Biology and Biotechnology "Charles Darwin", Università di Roma "La Sapienza", Italy; ${ }^{5}$ Department of Information Engineering, Università degli Studi di Firenze, Italy; ${ }^{6}$ Kayser Italia S.r.l., Livorno, Italy; ${ }^{7}$ Department of Experimental and Clinical Biomedical Sciences "Mario Serio", Università degli Studi di Firenze, Italy.

$\S$ These authors equally contributed to this study

${ }^{*}$ Corresponding author:

Matteo Lulli_matteo.lulli@unifi.it tel. +390552751329

Department of Experimental and Clinical Biomedical Sciences "Mario Serio", Università degli Studi di Firenze, viale Morgagni 50, 50134, Firenze, Italy 


\begin{abstract}
Astronauts on board the International Space Station (ISS) are exposed to the damaging effects of microgravity and cosmic radiation. One of the most critical and sensitive districts of their organism is the eye, and in particular the retina, so that more than half of them develops a complex of alterations designated as Spaceflight Associated Neuro-ocular Syndrome. We explored the cellular and molecular effects induced on human retinal pigment ARPE-19 cell line by their transfer to and three days stay on board the ISS in the context of an experiment funded by the Agenzia Spaziale Italiana (ASI). Treatment of cells on board ISS with the wellknown bioenergetic, antioxidant and antiapoptotic coenzyme Q10 was also evaluated. In the ground control experiment the cells were exposed to the same conditions as on the ISS, except for microgravity and radiation.

Transfer of ARPE-19 retinal cells to the ISS and their living on board for three days did not impact on cell viability or apoptosis but induced cytoskeleton remodeling consisting in vimentin redistribution from the cellular boundaries to the perinuclear area, underlining the collapse of the network of intermediate vimentin filaments under unloading conditions. Morphological changes endured by ARPE-19 cells grown on board the ISS were associated with changes in the transcriptomic profile related to cellular response to space environment, and were consistent with cell dysfunction adaptations. In addition, results obtained from ARPE-19 cells treated on board ISS with coenzyme Q10 showed its potential ability to increase cell resistance to damaging insults.
\end{abstract}

Keywords: retina; microgravity; radiations; retinopathy; space flight. 


\section{INTRODUCTION}

Space radiations and microgravity present in spacecraft and Space Stations in orbit around the Earth cause several time-dependent health alterations in astronauts both during their missions and after their return on the Earth. The most known, which are fluid shifts, loss of bone and muscle mass, cardiovascular imbalances, alterations in immunity, sleep interruption and circadian misalignment, have been described for more than two decades in a multitude of scientific reports and reviews [1-6]. At the cellular level, the main pathological effect of microgravity and cosmic radiation are structural alterations caused, among others, by free radical-mediated molecular damage [7-10].

Space exploration has now entered a new phase in which NASA, ESA and other national space agencies are working together to plan long-term space missions in order, for example, to create lunar bases and reach other space destinations such as Mars. At this stage, assessing the risk inherent in space missions and challenging the numerous obstacles to astronaut safety are increasingly important to consider in mission planning. In particular, since the microgravity and ionizing radiations inevitably present in the spacecraft environment represent two serious stress factors for astronaut health, the discovery of pharmacological countermeasures is obviously a mandatory prerequisite for mission planning $[11,12]$. A basic principle of pharmacological research is that the most valid rational basis for identifying tools capable of blocking or inhibiting a pathological process is the knowledge of its pathogenic mechanisms. Obviously, this principle applies to the variety of damages and health impairments known to be endured by astronauts during their long duration space missions.

The eye, and especially the retina, is severely sensitive to radiation and microgravity damage, to which retina eventually responds with apoptotic cell death $[9,13,10,14-18]$. About a decade ago, NASA reported that nearly $60 \%$ of about 300 astronauts returning on Earth after long-duration stay aboard the International Space Station (ISS) were affected by several neuro-ophthalmic alterations, including disc oedema, globe flattening, choroidal and retinal folds, hyperopic refractive error shifts, nerve fiber layer infarcts and visual acuity reduction [19], comprehensively termed Spaceflight Associated Neuro-ocular Syndrome (SANS) [2023]. Pathogenesis of SANS is not currently fully understood. The relevance of astronaut's visual impairment led NASA to consider vision as one of the top health risks for long-term space flight [24].

The ISS, where astronauts are exposed for several months to the space environment [25], offers a great opportunity to directly study astronaut health impairments but also to execute experiments aimed to unravel damage's pathogenesis even at the molecular level 
and to test the effectiveness of possible countermeasures, able to minimize damage and therefore to maximize the feasibility of the space missions.

In this paper, we report the impact exerted both on the cell structure and on the transcriptomic profile by the transfer of human retinal pigment epithelial ARPE-19 cells from the Earth to the ISS and their subsequent culture on board for three days. Effects of treatment of ARPE-19 cells with the coenzyme Q10 (CoQ10), by virtue of its well-known antioxidant and antiapoptotic abilities [26-29], were also reported. The results presented here were obtained in the context of the project "The Coenzyme Q10 (CoQ10) as Countermeasure for Retinal Damage on board the International Space Station: the CORM Project", funded by the Agenzia Spaziale Italiana (ASI) and belonging to a group of investigations of the ESA VITA (Vitality, Innovation, Technology, Ability) mission, which have been included in the ISS expedition $52 / 53$. To optimize the conditions for the experiment aboard the ISS, we previously carried out on-ground experiments revealing that a three-days stay in simulated microgravity (through a Rotating Wall Vessel bioreactor) leads ARPE-19 cells to undergo apoptosis, while X-rays induce a dose-dependent DNA damage accompanied by a DNA damage response (DDR) at telomeres, referred to as Telomere Dysfunction-Induced Foci (TIFs) accumulation [26].

\section{MATERIAL AND METHODS}

\section{Cell Culture}

The human retinal pigment epithelial ARPE-19 cells (ATCC, Manassas, VA, USA) were cultured in 50\% Dulbecco's Modified Eagles Medium (DMEM, Lonza, Basel, CH) and 50\% Ham's F12 Medium (Lonza) supplemented with 10\% fetal bovine serum (FBS, \# 35-015-CV; Corning, NY, USA), $100 \mathrm{U} / \mathrm{ml}$ penicillin-streptomycin (Lonza), $2 \mathrm{mM} \mathrm{L-glutamine} \mathrm{(Lonza)} \mathrm{in} \mathrm{a}$ humidified incubator at $37^{\circ} \mathrm{C}$ and $5 \% \mathrm{CO}_{2}$. ARPE-19 cells were frozen and shipped to the Kennedy Space Center (KSC), Space Station Processing Facility (SSPF) laboratories (Cape Canaveral, Florida, USA) months before the scheduled launch date. Two weeks before launch, cells were thawed and cultured in the KSC laboratories. For the experiment on board the ISS, ARPE-19 cells were seeded at a density of 20,000 cells $/ \mathrm{cm}^{2}$ on cell culture supports, i.e. Cyclic Olefin Copolymer (COC) ibiTreat plastic coverslips (IBIDI, Martinsried, Germany), which allow cell adhesion that is comparable to standard cell culture flasks, flexibility and good optical performance. Dedicated Experiment Units (EUs, KEU-ST, Kayser Italia, Livorno, Italy), which are electromechanical devices capable of executing cell culture protocols, qualified for flight to ISS, were used for the experiment on board ISS (Figure 1A-B). EUs contain five reservoirs: three were loaded with cell cultured media (50\% DMEM, 50\% Ham's F12, 10\% 
FBS, $100 \mathrm{U} / \mathrm{ml}$ Penicillin-Streptomycin, $2 \mathrm{mM} \mathrm{L-Glutamine,} \mathrm{25mM} \mathrm{Hepes} \mathrm{(Lonza))} \mathrm{in} \mathrm{presence}$ or absence of $10 \mu \mathrm{M}$ coenzyme Q10 (Sigma-Aldrich, St. Louis, USA) dissolved in a commercial vehicle to ensure cellular uptake (0.04\% Kolliphor P407 micro, Sigma-Aldrich); one with Dulbecco's phosphate buffered saline (DPBS, added with Ca2+/Mg2+, Lonza) for cell rinse prior to fixation; one with RNAlater (Ambion AM7020, MA, USA) as fixative solution. At day 1 after seeding, cell cultures on COC plastic coverslips were transferred into cell culture chambers of the EUs, which in turn were introduced into Experiment Containers KIC-SL (ECs) to compose the Experiment Hardware $(\mathrm{EH})$. EH were inserted into a passive temperaturecontrolled transportation container, named Biokit (Kayser Italia), containing phase change materials preheated at $27^{\circ} \mathrm{C}$. An iButton data logger were located on a $\mathrm{EH}$ to record the temperature every minute until the end of the experiment (recorded temperature profile is shown in Figure 1C). Three EHs were transferred to flight authorities (two containing cells not treated with CoQ10, and one containing cells treated with CoQ10) at day 2 after cell seeding. On August $14^{\text {th }}, 2017$, hardware was launched on a Dragon/Falcon 9 vector in the framework of the Space X CRS-12 mission at day 3 after seeding; it reached the ISS at day 5. On board the ISS, astronaut Paolo Nespoli manually inserted the EHs in the ESA Kubik incubator set at $37^{\circ} \mathrm{C}$, hence powering the EHs. Fluidic activations were automatically performed, allowing medium replacements at days 6,7 and 8 after seeding, and rinse and fixation at day 9. In particular, fixation with RNAlater occurred after 5 min from rinse with DPBS saline solution. In total, the EHs spent 72 hours inside the Kubik facility. Samples were then manually transferred into the MELFI (Minus Eighty Laboratory Freezer for ISS) refrigerator by astronaut Paolo Nespoli and stowed at $-80 \circ \mathrm{C}$ until their return to Earth on September 16. Temperature profile was downloaded from data logger, allowing to reproduce the same time/temperature profile to on ground control experiments. Once on ground, samples were thawed and retrieved from the EUs. COC plastic coverslips containing cells were sectioned with a scalpel while they were continuously submersed in RNAlater. 5 sections were obtained from each sample: 1 was subjected to apoptosis evaluation through TUNEL assay, 1 to vimentin immunofluorescence analysis, 3 to transcriptomic analysis. Images of cells were acquired by optical microscopy with an EVOS XL Core Imaging System (Thermo Fisher, USA).

\section{TUNEL}

DeadEnd Fluorometric TUNEL System kit (Promega, USA) was used to detect DNA breaks according to the manufacturer's protocol. Briefly, samples were fixed in $4 \%$ formaldehyde solution in PBS for $25 \mathrm{~min}$ at $+4^{\circ} \mathrm{C}$ and washed twice in PBS. Successively, cells were permeabilized with $0.2 \%$ Triton X- 100 in PBS for 5 min. After wash with PBS, samples were incubated in equilibration buffer from the staining kit for 10 min and then they were treated with staining reaction mix for $1 \mathrm{~h}$ at $37^{\circ} \mathrm{C}$ in humidified chamber protected from 
light. The reaction was arrested by 15 min incubation in $2 \times$ SSC. After wash with PBS, cells were stained with Hoechst 33242 dye (40,6-diamidino-2-phenylindole; ThermoFisher Scientific) for $10 \mathrm{~min}$ and mounted with ProLong Diamond Antifade Mountant (Thermo Fisher Scientific). For positive staining control, the slides were treated after permeabilization with DNase I (10 units $/ \mathrm{mL}$ ) for $10 \mathrm{~min}$. Samples were analyzed using a confocal microscope (Nikon TE2000 using EZ-C1 Software; Nikon Corp.), equipped with a 60XA/1.40 oil-immersion objective and digitally captured.

\section{Immunofluorescence}

Cells grown on the plastic coverslip and treated as previously described, were fixed for 5 min with ice cold acetone. Unspecific binding sites were blocked with PBS containing 3\% bovine serum albumin (BSA; Sigma-Aldrich, St. Louis, Missouri, US) for $1 \mathrm{~h}$ at room temperature. Then, cells were incubated overnight at $4^{\circ} \mathrm{C}$ with anti-vimentin antibody (\#MAB1681, Chemicon, Merck KGaA, Darmstadt, Germany) diluted 1:100 in PBS with 0.5\% BSA. After washing three times with PBS-0.5\% BSA, samples were then incubated for $1 \mathrm{~h}$ at room temperature in the dark with fluorescein isothiocyanate (FITC) conjugated secondary antibody [anti-mouse IgM (\#AP128F, Chemicon, Merck KGaA, Darmstadt, Germany) diluted 1:200]. Again, samples were washed three times and then mounted on glass slides using Fluoromount $^{\mathrm{TM}}$ aqueous mounting medium (Sigma-Aldrich St. Louis, Missouri, USA). Samples were evaluated by an epifluorescence microscope (Nikon, Florence, Italy) at 100x magnification and imaged by a HiRes IV digital CCD camera (DTA, Pisa, Italy).

\section{RNA isolation}

RNA extraction was performed using RNAqueous Total RNA Isolation kit (Ambion AM1912) following manufacturer instructions and eluting RNA in $40 \mu$ volume. Samples were vacuum-concentrated using DNA 120 Speedvac $\AA$ (ThermoSavant, USA) with low vacuum and no heating to avoid RNA fragmentation, manually checking samples every 5 minutes until volume reached $20 \mu \mathrm{l}$. The residual genomic DNA was removed by using DNA-free DNA Removal kit (Ambion AM1906) following manufacturer instructions. Isolated RNA and library preparation products were quantified using Qubit 3.0 (Invitrogen, USA) and quality was assessed by capillary electrophoresis using AATI Fragment Analyzer (Advanced Analytical Technologies, Inc., USA).

\section{Gene expression analysis}

Quantification of RNA expression was performed at Polo d'Innovazione di Genomica, Genetica e Biologia (Polo GGB, Siena, Italy) by RNAseq analysis. Total ribo-depleted cDNA libraries were prepared with SMARTer Stranded Total RNA-Seq Kit Pico Input Mammalian 
(Takara Bio USA, Inc.) according to manufacturer instructions. Indexed DNA libraries were normalized to a concentration of $4 \mathrm{nM}$ and then pooled in equal volumes to obtain a uniform reads distribution. The pool has been denatured and diluted to the final loading concentration of $1.4 \mathrm{pM}$ according to the Illumina protocol, with the addition of $1 \%$ spike in of PhiX Control v3 (Illumina) as sequencing control. To obtain a minimum of 20 million reads per sample, the pool has been sequenced using NextSeq 550 sequencer (Illumina) using a NextSeq 500/550 Mid Output v2 kit (150 cycles) (Illumina) to perform a paired end run 2X75 bp read length.

Raw reads for each sample were aligned to the human_g1k_v37 release of the GRCh37/hg19 human reference genome using the RNA-seq aligner STAR [30] (version 2.5.2b) with the default parameter settings. The following count-based differential expression analysis was performed using featureCounts [31] and DeSEQ2 [32]. More in detail, we counted reads mapped to each genomic meta-feature (that is, each gene) with featureCounts (version 1.5.1), using the default parameter settings and the Ensembl Gene Transfer Format (GTF) file for the appropriate reference genome (Ensembl release 82). The count matrix from featureCounts (where each row represents an Ensembl gene, each column a sequenced RNA library, and the values give the raw numbers of fragments that were uniquely assigned to the respective gene in each library) was subsequently converted into a DESeqDataSet object inside the $\mathrm{R}$ statistical environment. The following differential expression analysis, including the estimation of size factors (which takes into account differences in the sequencing depth of the samples), the estimation of dispersion values for each gene, and fitting a generalized linear model, was carried out by the DESeq function from the DeSEQ2 R package (release 3.3).

\section{Pathway analysis}

The data were analyzed using iPathwayGuide platform in the context of pathways obtained from the Kyoto Encyclopedia of Genes and Genomes (KEGG) database (Release 84.0+/10-26, Oct 17), gene ontologies from the Gene Ontology (GO) Consortium database (2017-Nov6), miRNAs from the miRBase (Release 21) and TARGETSCAN (Targetscan version: Mouse:7.1, Human:7.1) databases. iPathwayGuide scores pathways using the Impact Analysis method [33], which uses two types of evidence: i) the over-representation of DE genes in a given pathway and ii) the perturbation of that pathway computed by propagating the measured expression changes across the pathway topology. These aspects are captured by two independent probability values, pORA and pAcc respectively, that are then combined in a unique pathway-specific $p$-value. pORA expresses the probability of observing the number of $D E$ genes in a given pathway that is equal to or greater than the one observed by random chance. pAcc is calculated based on the amount of total gene accumulation measured in each pathway. Once accumulations of all gene number perturbations are computed, iPathwayGuide computes the total accumulation of the pathway as the sum of all absolute accumulations of 
the genes in a given pathway. The two types of evidence, pORA and pAcc, are combined into one final pathway score by calculating a p-value using Fisher's method, which is then rectified for multiple comparisons using false discovery rate (FDR) or Bonferroni corrections. Bonferroni correction, simpler and more conservative than FDR, reduces the false discovery rate by imposing a stringent threshold on each comparison adjusted for the total number of comparisons. The FDR correction has more power but only controls the family-wise false positives rate.

\section{RESULTS}

As previously described and shown in Figure 1, the CORM experiment was set up in the NASA KSC SSPF laboratory (Cape Canaveral, Florida, USA), where ARPE-19 cells, treated with CoQ10 or not, were seeded in a dedicated Experiment Unit (EU), which was then introduced into Experiment Containers (EC) to compose the Experiment Hardware (EH) [26] (Figure 1A). In turn, EH was included in the passive temperature-controlled transport container Biokit, which was launched to the ISS in the context of SpaceX CRS-12. Three EH were transferred to the ISS: two containing cells not treated with CoQ10, and one containing cells treated with CoQ10. Biokit's transfer to the ISS lasted three days, during which the cells underwent constant decrease in temperature up to about $26^{\circ} \mathrm{C}$. Once on board the ISS, EH were introduced inside the ESA KUBIK (incubator facility), set at $37^{\circ} \mathrm{C}$, allowing automatic execution of fluidic activations according to the programmed schedule (Figure 1B). After three days, the cells were fixed in RNAlater and $\mathrm{EH}$ were stored at $-80^{\circ} \mathrm{C}$ in the MELFI, where they were kept until its splashdown on ground. Once in our laboratories, still in freezing conditions, $\mathrm{EH}$ underwent final disassembling and samples were inspected. On ground ARPE-19 cell cultures were also prepared as control at normal gravity using similar $\mathrm{EH}$, following the temperature/time profile of the in-flight experiment (Figure 1C).

\section{Transferring ARPE-19 cells to ISS and culturing on board for three days do not} affect cell proliferation or apoptosis but severely modify vimentin organization. Some key cellular parameters we first evaluated after the flight are shown in the Figures 2 . The transfer of ARPE-19 cells (treated or not with CoQ10) to the ISS and the three days of incubation on board had no impact on their proliferation rate compared to ground controls. In both cases, the cells almost reached the confluence and did not reveal any signs of suffering (Figure 2A) nor underwent apoptosis assessed by TUNEL assay (Figure 2B). However, as shown in the representative images of immunofluorescence microscopy analysis in Figure 2C, the organization of the vimentin network underwent profound alterations consisting in a 
marked increase in its localization in the cellular perinuclear area and a concomitant redistribution from the cellular borders, which emphasized the collapse of the vimentin intermediate filament network.

Transferring ARPE-19 cells to ISS and culturing on board for three days dramatically modify transcriptome profile. We have then explored the possibility that spaceflight alters gene expression by analyzing ARPE-19 transcriptome profiles respect to ground controls through a next-generation sequencing technology, assuming a threshold of 0.05 for statistical significance ( $p$-value) and a change of expression of a log fold with an absolute value of at least 1 . Out of 23556 genes we analyzed, 5555 genes were differentially expressed (DE) after spaceflight with respect to the ground controls (Figure 3A and Supplementary S1). Among them, 3081 were up-regulated and 2474 were down-regulated. To predict the impact of ISS environment on ARPE-19 gene expression pathways, we used the iPathwayGuide to assess the possibility that a specific pathway was perturbed and that the subsequent genes in a pathway were significantly more perturbed respect to the previous ones. The results predicted that 99 pathways defined in the KEGG database were significantly affected (Figure 3B and Supplementary S2). The first ten pathways are shown in Table 1, and pathway diagrams containing coherent cascades and DE pathway genes are shown in Supplementary S3A-I. The most significantly impacted pathways were somehow related to cellular response to space environment adaptation/damage. The analysis of DE genes associated with perturbed pathways revealed a down-modulation of metabolic pathways, NGlycan biosynthesis, protein processing in the endoplasmic reticulum, p53 signaling, cellular senescence, mitophagy and steroid biosynthesis, and an up-modulation of MAPK, TGF-beta and Rap1 signaling (Figure 3C). To further explore the functional roles of the DE genes, we performed GO analysis (Figure 4 and Supplementary S4), which showed that transfer of ARPE-19 cells to ISS and their culturing for three days on board cause an alteration in response to unfolded protein, stress response of the endoplasmic reticulum and ion binding. The first five GO terms in biological processes, molecular functions and cellular components are listed in Table 2.

The presence of IncRNAs was screened among the DE genes, based on the IncRNAs noted on the HUGO gene nomenclature committee website (HGNC), which included a total of 4118 IncRNAs (http://www.genenames.org/cgi-bin/statistics) (Supplementary S5). Expression of 255 IncRNAs was deregulated in ARPE-19 cells cultured on board ISS; 208 were upregulated and 47 were downregulated (top ten up- and downregulated in Table 3). To predict active micro-RNAs (miRNAs) in ARPE-19 cells cultured on board ISS compared to ground control, the DE genes were also analyzed to disclose the presence of enriched DE targets of the miRNAs. For a given miRNA, the analysis computed the ratio between the 
number of DE targets and all differentially expressed targets and compared it with the ratio of all targets expressed downwards with all targets. It allowed to calculate the probability of observing a greater number of differentially downregulated target genes for a given miRNA only by chance. Out of a total 366 screened miRNAs, 19 miRNAs achieved significant expression values, as described in Fig. 5 and Supplementary S6, with miR-296-5p, miR-494$3 p$ and miR-128-3p showing the best $p$-value.

We have then evaluated the role of CoQ10 treatment on gene expression of ARPE-19 cultured on board ISS. Assuming a threshold of 0.05 for $p$-value and of 0.4 for fold change, out of 26694 genes we analyzed, we found 153 DE genes in cells treated with CoQ10 with respect to untreated controls (Figure 6A and Supplementary S7). Among these DE genes, 81 were up-regulated and 72 were down-regulated in the spaceflight ARPE-19 cells. iPathwayGuide analysis predicted that 22 pathways defined in the KEGG database were significantly affected (Figure 6B and Supplementary S8), among which type I diabetes mellitus, HIF-1 signaling, ferroptosis and Notch signaling pathways are the most significantly impacted.

\section{DISCUSSION}

In the present study, we investigated the impact of the space environment on human retinal pigment epithelial cells. For this purpose, ARPE-19 cells were transferred and cultured for three days onboard ISS; in addition, cells were cultured in presence of not of the wellknown bioenergetics, antioxidant and antiapoptotic agent CoQ10. As control, ARPE-19 cells were cultured on-ground under normal $1 \mathrm{~g}$ condition.

Previous on ground experiments on ARPE-19 cells using microgravity simulators led to contrasting results. We previously showed that three days of simulated microgravity obtained by the Rotating Wall Vessel bioreactor (RWV) increased the activity of caspase 3/7 and determined a reduction of mitochondrial transmembrane potential, both evidences of apoptosis induction [26]. Instead, using a different microgravity simulator (Random Positioning Machinery, RPM), Corydon and colleagues did not report apoptotic events in ARPE19 cells cultured for 5-10 days in simulated microgravity condition [34]. We found that the transfer of ARPE-19 cells from Cape Canaveral to the ISS and three day incubation on board did not affect their proliferation rate nor led them to apoptosis with respect to on ground controls. These results could be explained by differences existing between microgravity simulated by the available simulator devices and between simulated microgravity and real microgravity onboard ISS; the use of these devices, and the impact of flight hardware on cellular physiology, are currently debated $[35,36]$. Cells living onboard ISS are simultaneously subjected to 
microgravity and space radiation, which usually do not occur in on-ground simulation experiments, where the two treatments are administered separately. In fact, the constant threat to the integrity of astronauts represented by space radiations, causing damage to DNA directly or through the production of free radicals, and microgravity, hampering DNA repair, have raised the opportunity to explore the question of potential synergies between microgravity and radiation, both in space and in earthy analogues. Results have been conflicting, which indicated the need to perform experiments in the space environment [37]. Furthermore, in our experiment the three-day culturing of ARPE-19 cells on board the ISS has been preceded by a five-days permanence into the Biokit at a relatively low temperature (up to about $26^{\circ} \mathrm{C}$ ). It has been demonstrated that hypothermia elicits a strong expression of cold shock proteins in mammalian retinal cells, including retinal pigment epithelial cells [38], where they may act as protective factors against damaging insults and prevent apoptosis [39]. Finally, it should be noted that ARPE-19 cells were cultured inside the KUBIK on board the ISS for three days, however, due to the transfer from on ground to ISS, the cells underwent microgravity condition several hours before entering the KUBIK, which could affect adaptive behavior.

Cytoskeletal disorganization and significant changes in the expression of its main components, actin, tubulin and vimentin, both at phenotypic and genotypic levels have been observed in various cell type subjected to altered gravity conditions [40-43] and the complete destruction of the microtubule network was observed in Jurkat cells following exposure to real microgravity [44]. Similarly, in endothelial cells, a significant reduction in actin fibers was observed in response to simulated microgravity [45] and a significant increase in stress fibers following hypergravity [46]. ARPE-19 cells cultured for $5 \mathrm{~d}$ and $10 \mathrm{~d}$ on RPM revealed the appearance of two major phenotypes, i.e. adherent monolayers and 3D multicellular spheroids [34]. In addition, the reduction of F-actin filaments in favors of F-actin structures at the cell boundaries, and the downregulation of beta-actin, beta-tubulin, keratin 8 , vimentin, laminin subunit beta 2, integrin beta 1, integrin beta 3, collagen 4 gene expression have been revealed, suggesting that simulated microgravity induces a reduced capability to retain adhesion and stiffness of exterior structures in the ARPE-19 cells. Other cell types differ from ARPE-19 cells in their cytoskeleton-related response to microgravity. For example, RPM induces upregulation of beta actin gene expression in MCF-7 breast carcinoma [47] and in human primary chondrocytes [48], and upregulation of vimentin gene expression in FLG 29.1 leukemic cells [46] and ML-1 follicular thyroid carcinoma cells [49]. Here, we revealed a dramatic change of cytoskeletal vimentin distribution in ARPE-19 cultured for three-days onboard ISS. Vimentin is a cytoskeletal protein responsible for maintaining cellular integrity, being the main constituent of intermediate filaments, which provide structural support for the cytoplasm and play an essential role in the response to mechanical stresses, such as gravity. 
Recently, it has been demonstrated that the vimentin network maintains the resilience of cytoplasm, and enhances cytoplasmic mechanical strength and toughness under dynamic deformation, through poroelastic and viscoelaxic relaxation [50]. As a result, vimentin networks may reduce the risk of cell damage undergoing major deformation events. In samples of ARPE-19 cells exposed for three days to real microgravity, the results we obtained by immunofluorescence analysis showed a deregulated vimentin cellular localization, mostly concentrated in the perinuclear region. The different distribution of vimentin in cells exposed to microgravity, compared to the control, denotes a change in the shape of the cells and suggests an alteration in the capability of cells to interact with others and with extracellular matrix components.

The first observations that microgravity altered gene expression appeared more than twenty years ago but, since the most obvious detrimental effect of long-lasting space flights on astronauts was the strong atrophy of their musculoskeletal system, they were mainly focused on gene expression in bone and muscle cells [51,52]. Subsequently, several studies have highlighted the impact of the spatial environment on gene expression in different tissues, but a real milestone for high-performance studies of the expression of entire panels of genes in response to spatial radiation and to microgravity have been the recent genomic and proteomic approaches. Apparently, understanding the variations of gene expression in their maximum amplitude is the key to unravelling the pathogenesis of damages endured by astronauts during spaceflight and, ultimately, to find effective countermeasures. This study investigated gene expression changes in ARPE-19 cells transferred and cultured for three days onboard ISS. We found a considerable number of upregulated and downregulated DE genes. KEGG analysis, impact analysis and GO annotation revealed that several relevant biological pathways and cellular functions were affected by space flight. Among them, protein processing in endoplasmic reticulum (ER) is one of the most impacted, which is also justified by vimentin network alterations we have reported. ER is a complex endomembrane system, where proteins are folded and transported to distinct cellular districts. Corrected folded proteins are packaged into transport vesicles and shuttled into the Golgi system. Misfolded proteins are retained within ER, coupled with chaperones and, if terminally misfolded, subjected to ER-associated degradation. ER accumulation of misfolded proteins determines ER stress and evokes the unfolded protein response (UPR), which aim is to inhibit protein translation, to degrade misfolded proteins, to increase chaperones production or, eventually, to induce apoptosis. ER stress and UPR have been correlated to microgravity in different cell types [53-55]. We found that the majority of DE genes involved in this pathway were downregulated in ARPE-19 cells cultured onboard ISS. However, some members of the 70 kilodalton heat shock proteins (HSP70) gene family (HSPA1A, HSPA8 and HSPA2 in particular) and E3 ligase Parkin (PARK2) gene, involved in chaperone and E3 ubiquitin ligase 
activity, respectively, were significantly upregulated. Interesting studies have been recently conducted in human endothelial cells (HUVECs). It has been reported that 4 days of HUVEC culture in RWV simulated microgravity induced cell stress and determined HSP70 upregulation, probably sustaining the early phases of the dynamic adaptation of HUVECs to microgravity, while HSP70 silencing impaired cell survival [56]. In addition, they demonstrated a reduction of mitochondrial function and mitochondrial content, and that mitophagy contributes to this adaptation that culminates in a thrifty phenotype [41]. In addition to its role in ER metabolism, Parkin is a central regulator of mitophagy, another pathway significantly impacted in ARPE-19 cells cultured onboard ISS. Deleterious byproducts or oxidative stress lead to mitochondrial dysfunction, which, if the damage is unrepairable, culminates in mitochondria recognition and targeting for degradation through the autophagic process termed mitophagy. Multi-omicHs analysis recently identified mitochondrial dysregulation as a central biological hub for spaceflight pathophysiological effects [57]. Parkin acts in this context, recognizing altered proteins on the mitochondrial outer membrane and mediating damaged mitochondria clearance. Microphthalmia-associated transcription factor (MITF) is another gene belonging to the mitophagy pathway that resulted upregulated in space ARPE-19 cells. MITF is a transcription factor responsible for retinal pigment epithelial cell development and functions. MITF plays a protective role against oxidative stress in ARPE-19 cells, where it upregulates antioxidant gene expression and mitochondrial biogenesis, mainly through peroxisome proliferator-activated receptor-gamma coactivator 1 alpha (PGC1a) and nuclear factor erythroid 2-related factor 2 (NRF2) upregulation [58].

TGF-beta and p53 signaling were deregulated in ARPE-19 cells cultured onboard the ISS. While most of DE genes involved in TGF-beta signaling were upregulated, the opposite scenario was for p53 signaling. Through a system biology approach evaluating rodent transcriptomic data from GeneLab (genelab.nasa.gov), TGF-beta and p53 were identified as the most prevalent pan-tissue signaling pathways activated in response to microgravity [59]. p53 is a major contributor of cell cycle arrest, DNA repair and apoptosis induction in response to genotoxic or non-genotoxic damages. p53 signaling has been found frequently activated in response to microgravity. However, depending on cell types and culture conditions, heterogeneous responses have been observed. For example, p53 pathway activation has been recently described in macrophages undergoing spaceflight or RWV simulated microgravity [60] and in lung cancer cells cultured with RPM [61], while inhibition of p53 signaling under 3D-clinostat simulated microgravity was found in hepatoblastoma cells. In ARPE-19 cells cultured onboard ISS, we detected that several p53-transactivated proapoptotic genes (e.g. BAX, PMAIP1, BBC3), and of genes involved in cell cycle arrest (e.g. $C D K N 1 A, G A D D 45 A, G A D D 45 B)$ and DNA repair and damage prevention (DDB2, GADD45A, GADD45B, SESN2) were downregulated. Along the same line, cellular senescence pathway 
genes, and in particular most of the genes coding senescence-associated secretory phenotype (SASP) factors, e.g. IL1A, CXCL8, SERPINE1 and IGFBP3, were significantly downregulated. On the contrary, the gene coding for the SASP TGF-beta factor was upregulated. TGF-beta arises as a master regulator that coordinates systemic response to microgravity at multiple biological scales [59]. TGF-beta is a well-known cytokine that belongs to a family of several protein members, including bone morphogenic proteins (BMPs), growth differentiation factors, activinins, inhibins, all of them regulating a wide spectrum of cellular functions such as proliferation, apoptosis, differentiation and immune response. TGF-beta plays a major role in RPE cells, in particular in a dynamic transition along a well differentiated, polarized epithelial to mesenchymal cells, that has been defined as RPE dysfunction [62]. Functional RPE cells form a single layer of polarized cells located between photoreceptors and choroid. RPE correct differentiation and polarization are essential for proper functions. Indeed, while epithelial feature guarantees RPE physiological homeostasis in the retina, different mechanisms have been found to be involved in RPE epithelial to mesenchymal transition (EMT) and mesenchymal to epithelial transition (MET), hindering their functions. Microgravity has been previously demonstrated to trigger EMT in different cell types $[60,63]$. TGF-beta signaling is one of the most potent EMT inductors, and therefore a major contributor of RPE dysfunction. High levels of vitreal TGF-beta have been detected in proliferative vitreoretinopathy patients, a pathological condition where EMT of RPE plays an essential pathogenic role [64], which led to evaluate inhibitors of TGF beta signaling, able to counteract RPE EMT, for treatment of proliferative retinal diseases such as proliferative vitreoretinopathy [65]. The Hippo signaling pathway is closely interconnected with that of TGF-beta, and resulted significantly affected in ARPE-19 cells cultured on board ISS. This pathway is a regulator of RPE cell differentiation, other to be involved in RPE EMT [66]. Connective tissue growth factor (CTGF) gene codes for a cysteine-rich extracellular matrix protein that acts downstream of TGF-beta and Hippo signaling pathways [67,68]. CTGF is one of the higher overexpressed genes in ARPE-19 cells cultured on board ISS. It has been hypothesized that its binding to various cell surface receptors, including integrin receptors, heparan sulfate proteoglycans and low-density lipoprotein receptor-related proteins, enables CTGF to regulate key cellular functions, such as cell adhesion, proliferation, chemotaxis, differentiation, survival, and ECM components production [69]. A previous study indicated that CTGF increases the migratory ability of RPE cells, and that it is a major mediator of retinal fibrosis. In addition, it is has been recently demonstrated that the Hippo pathway is severely altered in a murine model of retinal degeneration, in which CTGF undergoes a marked upregulation [68].

Analysis of DE genes revealed a deregulation in the expression and activity of IncRNAs and microRNAs, respectively, in ARPE-19 cells. LncRNAs are defined as transcripts longer than 200 nucleotides that generally lack protein-coding potential and can be processed like 
mRNAs, i.e. spliced and polyadenylated. LncRNAs are involved in several cellular physiological processes such as adaptation to stresses, cell differentiation, maintenance of pluripotency and apoptosis. The correct balance of IncRNA levels is crucial for the maintenance of cellular equilibrium, and their dysregulation is associated to many disorders [70]. Variations of IncRNA expression have been documented in different cell types exposed to simulated microgravity [71-73]. Here, we demonstrated that real microgravity alters a panel of IncRNAs in ARPE-19 cells, most of which were upregulated. Considering the top 10 upand down-regulated IncRNAs, the only involved to date in retinal cells physiology is $H 19$, in particular in retinal cell death and in the inflammatory response of ARPE-19 cells exposed to hyperglycemia [74]. We detected a strong downregulation of H19 expression in ARPE-19 cells cultured onboard ISS, and interestingly, it has been reported that $H 19$ downregulation correlates with TGF-beta mediated EMT in retinal endothelial cells [75]. On the same line of evidence, other two IncRNAs downregulated in ARPE-19 cells cultured onboard ISS, namely SLC7A11-AS1 and LUCAT1, are reported to be involved in the EMT process [76,77]. MicroRNAs are small single strand non-coding RNA, whose functions in RNA silencing and post-transcriptional regulation of gene expression are well known. A recent milestone study identified a spaceflight-associated microRNA signature in response to simulated, shortduration and long-duration spaceflight, and simulated deep space radiation conditions [78]. In addition, the panel of spaceflight-associated microRNA has been predicted to interfere with cell signaling and metabolic pathways, partly through interaction with TGF-beta signaling [59]. Some microRNAs we identified to be activated in ARPE-19 cells cultured on board ISS are involved in cell response to spaceflight. Among them, hsa-miR-296 expression is affected in HUVEC cultured in a 3D clinostat to simulate microgravity, and it influences cell proliferation and vascular function in microgravity conditions [79]. hsa-miR-494 is upregulated in $\mathrm{C} 2 \mathrm{C} 12$ myoblast cells subjected to clinorotation conditions and in osteoblasts isolated from tailsuspended rats, and this upregulation correlates with inhibition of osteoclast differentiation [80]. Relevant, other microRNAs we identified are involved in the EMT process. For example, hsa-miR-145-3p, hsa-miR-324-5p and hsa-miR142-5p act as inhibitors of EMT in different cell types [81-84]; on the contrary, high expression and activation of hsa-miR 483-3p, hsa-miR128-3p are involved in EMT induction [85,86].

Coenzyme Q10 is an essential cofactor of the electron transport chain. It is endowed with well-known protective actions in respect to different types of damages on various cell types, including those of the retina, which mainly depends on its free radical scavenger ability and on the regulation of mitochondrial permeability transition pore [29]. It is known that choroid/retina levels of endogenous CoQ10 decrease with aging concomitantly with progression of apoptosis-related retinal diseases [87]. We previously demonstrated the efficacy of topical administration (via eye drops) of CoQ10 to protect retinal cell layers 
commissioned to apoptosis by a variety of noxious stimuli $[27,28]$. On these bases, we tested the role of CoQ10 treatment on modulating transcriptome of ARPE-19 cells cultured onboard ISS. We found a limited number of DE genes, which were subjected to KEGG and impact analyses. Among the most impacted biological pathways affected following CoQ10 treatment, we point on HIF-1 signaling and ferroptosis. HIF-1 is a well-known transcription factor discovered by virtue of its role as master regulator of oxygen homeostasis. Besides, HIF-1 signaling is involved in numerous cellular responses to a plethora of environmental stresses. Interestingly, it has been demonstrated that, regardless of its function of transcription factor, HIF-1 has a protective role through its localization into mitochondria where it reduces ROS level and reverses mitochondrial damage [88]. Previous studies revealed that microgravity affects HIF-1 pathway activation. Wang et al demonstrated that 28 days of simulated microgravity induced oxidative stress and activation of HIF-1 and a panel of its downstream targets in rat hippocampus [89], while Vogel et al demonstrated that HIF-1a and HIF-1dependent transcripts were differentially regulated in altered gravity on monocytes and macrophages during parabolic flight and suborbital ballistic rocket campaigns, whereas HIF1-dependent gene expression adapted after 5 min microgravity [90]. Ferroptosis is a peculiar type of iron-dependent cell death, firstly characterized in 2012, which results from accumulation of toxic levels of lipid reactive oxidative species [91]. Increased intracellular iron accumulation is a classical ferroptosis activator, directly linked to the failure of the glutathione (GSH)-dependent antioxidant defenses. Cellular iron metabolism includes absorption of the complex ferritin-Fe3+ by transferrin receptor (TFRC), and it has been demonstrated that decreasing iron utilization may increase the sensitivity to ferroptosis [92]. GSH production is sustained by activity of the amino acid antiporter SLC7A11/xCT/system xc-, which guarantees the exchanging of extracellular cystine (which, in turn, generated cysteine, a limiting precursor for GSH synthesis) for intracellular glutamate. It is known that inhibition of the system xccauses GSH depletion to trigger ferroptosis [93]. A direct involvement of CoQ10 in ferroptosis has been recently disclosed. It is known that the reduced form of CoQ10 is a potent antioxidant that counteract lethal lipid peroxidation, thus acting as a ferroptosis suppressor. In particular, the NADH-dependent oxidoreductase FSP1 converts non-mitochondrial oxidized to reduced CoQ10, furnishing a GSH-independent protective axis to counteract membrane lipids peroxidation [94]. Beside this role, we revealed that CoQ10 treatment of ARPE-19 cells cultured on board ISS determines a reduction of TFRC and an induction of SLCTA11 gene expression, corroborating the evidence that CoQ10 acts as a direct ferroptosis inhibitor, and bona fide increases ferroptosis resistance through reducing iron cellular uptake and enhancing cystine uptake.

In conclusion, our results indicate that the transfer of cultured ARPE-19 human retinal cells to ISS and their three-day living on board severely alter cytoskeleton morphology and 
transcriptome profile, particularly indicating the emergence of a cellular dysfunction (Figure 7). Most likely, these two events strongly contribute to the pathogenesis of retina injuries suffered by astronauts during space flights, and suggest a rational basis for finding effective countermeasures. Treatment of ARPE-19 cells with CoQ10 on board reveals its potential ability to increase cell resilience towards harmful agents of the space environment.

\section{DECLARATIONS}

\section{Funding}

Project coordinated and funded by the Italian Space Agency (ASI, grant 2016-6-U0 to M.L.) for Space Station experimentation within the national ISS utilization rights. The authors thank Fondazione Cassa di Risparmio Di Firenze (Italy) for financial support.

\section{Conflicts of interest/Competing interests}

Authors declare no conflicts of interest/competing interests.

\section{Availability of data and material}

All the data and material used are available in the author's labs.

Code availability (software application or custom code)

Not applicable

\section{Ethics approval}

Not applicable

\section{Consent to participate}

Not applicable

\section{Consent for publication}

Not applicable 


\section{LEGEND TO FIGURES}

Figure 1 - A) The hardware of CORM experiment: the Experimental Units (EU) were integrated into Experimental Container to compose the Experiment Hardware (EH), which were placed inside the Biokit transportation container that was launched to the ISS. Once onboard ISS, the EH were inserted into the ESA KUBIK incubator in the ISS Columbus module. B) Schematic fluidic of the EU. C) iButton data logger-derived temperature profile of the CORM experiment.

Figure 2 - A) Representative photomicrographs obtained with phase contrast microscopy in ARPE-19 cell pre-launch and post-flight. B) Evaluation of apoptosis in ARPE-19 cells by TUNEL fluorescent staining. Image size: 250x250 um. C) Evaluation of vimentin cellular localization in ARPE-19 cells by immunofluorescence. Image size: 100x100 um.

Figure 3 - A) The 5555 significantly DE genes (shown in red) are represented in the Volcano plot in terms of their measured expression change (x-axis) and the significance of the change (y-axis). The significance is represented in terms of the negative log (base 10) of the $p$-value, so that genes that are more significant are plotted higher on the $y$-axis. The dotted lines represent the thresholds used to select the DE genes: 1 for expression change and 0.05 for significance. B) Pathways perturbation vs over-representation: the pathways are plotted in terms of the two types of evidence computed by iPathwayGuide: over-representation is on the $x$-axis (pORA) and the total pathway accumulation is on the $y$-axis (pAcc). Each pathway is represented by a single dot, with significant pathways shown in red, non-significant in black, and the size of each dot is proportional to the size of the pathway it represents. Both $p$-values are shown in terms of their negative log (base 10) values. C) Differentially expressed pathway genes of the most significant impacted pathways. Mean value and SD are graphed in red.

Figure 4 - GO analysis of DE genes; top ten GO terms in biological processes (green), molecular functions (red) and cellular components (blue) category are listed.

Figure 5 - A) Significant differences in specific miRNA expression plotted in term of the two types of evidence computed by iPathwayGuide: the $x$-axis $(-\log 10(p v))$ representing the $p$-value based on the total number of DE target genes versus the total number of target genes and the $y$-axis $(-\log 10(p v n))$ is the $p$-value based on the number of downwardly expressed DE targets versus the total number of DE miRNA targets. Each miRNA is represented by a single dot, with significant miRNAs shown in red, non-significant in black, and the size of each dot is proportional to the size of the number of target genes for that miRNA relative to other 
ones. B) List of the 19 miRNAs which reached significant expression values, depicting the number of DE target genes that are under-expressed (in blue) or overexpressed (in red).

Figure 6 - A) All 153 significantly DE genes (showed in red) are represented in the Volcano plot in terms of their measured expression change (x-axis) and the significance of the change (y-axis), as for Figure $3 A$. The dotted lines represent the thresholds used to select the DE genes: 0.4 for expression change and 0.05 for significance. B) Pathways perturbation vs over-representation. Each pathway is represented by a single dot, with significant pathways shown in red, non-significant in black, and the size of each dot is proportional to the size of the pathway it represents. Both p-values are shown in terms of their negative log (base 10) values.

Figure 7 - Space flight effects on ARPE-19 cells. 


\section{References}

1. Demontis GC, Germani MM, Caiani EG, Barravecchia I, Passino C, Angeloni D (2017) Human Pathophysiological Adaptations to the Space Environment. Frontiers in physiology 8:547.

2. Strollo F, Gentile S, Strollo G, Mambro A, Vernikos J (2018) Recent Progress in Space Physiology and Aging. Frontiers in physiology 9:1551.

3. Stavnichuk M, Mikolajewicz N, Corlett T, Morris M, Komarova SV (2020) A systematic review and meta-analysis of bone loss in space travelers. NPJ microgravity 6:13.

4. Norsk P (2020) Adaptation of the cardiovascular system to weightlessness: Surprises, paradoxes and implications for deep space missions. Acta Physiol (Oxf) 228 (3):e13434.

5. Crucian BE, Chouker A, Simpson RJ, Mehta S, Marshall G, Smith SM, Zwart SR, Heer M, Ponomarev S, Whitmire A, Frippiat JP, Douglas GL, Lorenzi H, Buchheim JI, Makedonas G, Ginsburg GS, Ott CM, Pierson DL, Krieger SS, Baecker N, Sams C (2018) Immune System Dysregulation During Spaceflight: Potential Countermeasures for Deep Space Exploration Missions. Front Immunol 9:1437.

6. Grigoriev AI, Bugrov SA, Bogomolov VV, Egorov AD, Polyakov VV, Tarasov IK, Shulzhenko EB (1993) Main medical results of extended flights on space station Mir in 1986-1990. Acta astronautica 29 (8).

7. Bradbury P, Wu H, Choi JU, Rowan AE, Zhang H, Poole K, Lauko J, Chou J (2020) Modeling the Impact of Microgravity at the Cellular Level: Implications for Human Disease. Front Cell Dev Biol 8:96.

8. Beck M, Moreels M, Quintens R, Abou-El-Ardat K, El-Saghire H, Tabury K, Michaux A, Janssen A, Neefs M, Van Oostveldt P, De Vos WH, Baatout S (2014) Chronic exposure to simulated space conditions predominantly affects cytoskeleton remodeling and oxidative stress response in mouse fetal fibroblasts. Int J Mol Med 34 (2):606-615.

9. Mao XW, Boerma M, Rodriguez D, Campbell-Beachler M, Jones T, Stanbouly S, Sridharan V, Nishiyama NC, Wroe A, Nelson GA (2018) Combined Effects of Low-Dose Proton Radiation and Simulated Microgravity on the Mouse Retina and the Hematopoietic System. Radiation research.

10. Mao XW, Pecaut MJ, Stodieck LS, Ferguson VL, Bateman TA, Bouxsein M, Jones TA, Moldovan M, Cunningham CE, Chieu J, Gridley DS (2013) Spaceflight environment induces mitochondrial oxidative damage in ocular tissue. Radiation research 180 (4):340-350.

11. Kast J, Yu Y, Seubert CN, Wotring VE, Derendorf H (2017) Drugs in Space: Pharmacokinetics and Pharmacodynamics in Astronauts. European journal of pharmaceutical sciences : official journal of the European Federation for Pharmaceutical Sciences 109S. 
12. Eyal S, Derendorf H (2019) Medications in Space: In Search of a Pharmacologist's Guide to the Galaxy. Pharm Res 36 (10):148.

13. Mao XW, Boerma M, Rodriguez D, Campbell-Beachler M, Jones T, Stanbouly S, Sridharan V, Wroe A, Nelson GA (2018) Acute Effect of Low-Dose Space Radiation on Mouse Retina and Retinal Endothelial Cells. Radiation research 190 (1):45-52.

14. Zhao HW, Zhao J, Hu LN, Liang JN, Shi YY, Nie C, Qiu CY, Nan XS, Li YX, Gao FL, Liu $Y$, Dong $Y$, Luo L (2016) Effect of long-term weightlessness on retina and optic nerve in tailsuspension rats. International journal of ophthalmology 9 (6):825-830.

15. Tombran-Tink J, Barnstable CJ (2006) Space flight environment induces degeneration in the retina of rat neonates. Advances in experimental medicine and biology 572:417-424.

16. Tombran-Tink J, Barnstable CJ (2005) Space shuttle flight environment induces degeneration in the retina of rat neonates. Gravitational and space biology bulletin : publication of the American Society for Gravitational and Space Biology 18 (2):97-98.

17. Roberts JE, Kukielczak BM, Chignell CF, Sik BH, Hu DN, Principato MA (2006) Simulated microgravity induced damage in human retinal pigment epithelial cells. Molecular vision 12:633-638.

18. Mader TH, Gibson CR, Caputo M, Hunter N, Taylor G, Charles J, Meehan RT (1993) Intraocular pressure and retinal vascular changes during transient exposure to microgravity. American journal of ophthalmology 115 (3):347-350.

19. Mader TH, Gibson CR, Pass AF, Kramer LA, Lee AG, Fogarty J, Tarver WJ, Dervay JP, Hamilton DR, Sargsyan A, Phillips JL, Tran D, Lipsky W, Choi J, Stern C, Kuyumjian R, Polk JD (2011) Optic disc edema, globe flattening, choroidal folds, and hyperopic shifts observed in astronauts after long-duration space flight. Ophthalmology 118 (10):2058-2069.

20. Mader TH, Gibson CR, Otto CA, Sargsyan AE, Miller NR, Subramanian PS, Hart SF, Lipsky W, Patel NB, Lee AG (2017) Persistent Asymmetric Optic Disc Swelling After LongDuration Space Flight: Implications for Pathogenesis. Journal of neuro-ophthalmology : the official journal of the North American Neuro-Ophthalmology Society 37 (2):133-139.

21. Zhang LF, Hargens AR (2018) Spaceflight-Induced Intracranial Hypertension and Visual Impairment: Pathophysiology and Countermeasures. Physiological reviews 98 (1):59-87.

22. Lee AG, Mader TH, Gibson CR, Tarver W (2017) Space Flight-Associated Neuro-ocular Syndrome. JAMA ophthalmology 135 (9):992-994.

23. Lee AG, Mader TH, Gibson CR, Tarver W, Rabiei P, Riascos RF, Galdamez LA, Brunstetter T (2020) Spaceflight associated neuro-ocular syndrome (SANS) and the neuroophthalmologic effects of microgravity: a review and an update. NPJ microgravity 6:7.

24. Tymko MM, Boulet LM, Donnelly J (2017) Intracranial pressure in outer space: preparing for the mission to Mars. J Physiol 595 (14):4587-4588. 
25. Alwood JS, Ronca AE, Mains RC, Shelhamer MJ, Smith JD, Goodwin TJ (2017) From the bench to exploration medicine: NASA life sciences translational research for human exploration and habitation missions. NPJ microgravity 3:5.

26. Lulli M, Cialdai F, Vignali L, Monici M, Luzzi S, Cicconi A, Cacchione S, Magi A, Di Gesualdo F, Balsamo M, Vukich M, Neri G, Donati A, Capaccioli S (2019) The Coenzyme Q10 (CoQ10) as Countermeasure for Retinal Damage Onboard the International Space Station: the CORM Project | SpringerLink. Microgravity Science and Technology 30 (6):925-931.

27. Lulli M, Witort E, Papucci L, Torre E, Schiavone N, Dal Monte M, Capaccioli S (2012) Coenzyme Q10 protects retinal cells from apoptosis induced by radiation in vitro and in vivo. J Radiat Res 53 (5):695-703.

28. Lulli M, Witort E, Papucci L, Torre E, Schipani C, Bergamini C, Dal Monte M, Capaccioli S (2012) Coenzyme Q10 instilled as eye drops on the cornea reaches the retina and protects retinal layers from apoptosis in a mouse model of kainate-induced retinal damage. Investigative ophthalmology \& visual science 53 (13):8295-8302.

29. Papucci L, Schiavone N, Witort E, Donnini M, Lapucci A, Tempestini A, Formigli L, ZecchiOrlandini S, Orlandini G, Carella G, Brancato R, Capaccioli S (2003) Coenzyme q10 prevents apoptosis by inhibiting mitochondrial depolarization independently of its free radical scavenging property. The Journal of biological chemistry 278 (30):28220-28228.

30. Dobin A, Davis CA, Schlesinger F, Drenkow J, Zaleski C, Jha S, Batut P, Chaisson M, Gingeras T (2013) STAR: ultrafast universal RNA-seq aligner. Bioinformatics (Oxford, England) 29 (1).

31. Liao Y, Smyth G, Shi K (2014) featureCounts: an efficient general purpose program for assigning sequence reads to genomic features. Bioinformatics (Oxford, England) 30 (7).

32. Love M, Huber W, Anders I (2014) Moderated estimation of fold change and dispersion for RNA-seq data with DESeq2. Genome biology 15 (12).

33. Ashan S, Drăghici S (2017) Identifying Significantly Impacted Pathways and Putative Mechanisms With iPathwayGuide. Current protocols in bioinformatics 57.

34. Corydon TJ, Mann V, Slumstrup L, Kopp S, Sahana J, Askou AL, Magnusson NE, Echegoyen D, Bek T, Sundaresan A, Riwaldt S, Bauer J, Infanger M, Grimm D (2016) Reduced Expression of Cytoskeletal and Extracellular Matrix Genes in Human Adult Retinal Pigment Epithelium Cells Exposed to Simulated Microgravity. Cellular physiology and biochemistry : international journal of experimental cellular physiology, biochemistry, and pharmacology 40 (1-2).

35. Krüger M, Pietsch J, Bauer J, Kopp S, Carvalho DTO, Baatout S, Moreels M, Melnik D, Wehland M, Egli M, Jayashree S, Kobberø SD, Corydon TJ, Nebuloni S, Gass S, Evert M, Infanger M, Grimm D (2019) Growth of Endothelial Cells in Space and in Simulated Microgravity - a Comparison on the Secretory Level. Cellular physiology and biochemistry : 
international journal of experimental cellular physiology, biochemistry, and pharmacology 52 (5).

36. Wuest SL, Richard S, Kopp S, Grimm D, Egli M (2015) Simulated microgravity: critical review on the use of random positioning machines for mammalian cell culture BioMed research international 2015.

37. Moreno-Villanueva M, Wong M, Lu T, Zhang Y, Wu H (2017) Interplay of space radiation and microgravity in DNA damage and DNA damage response. NPJ microgravity 3.

38. Larrayoz IM, Rey-Funes M, Contartese DS, Rolón F, Sarotto A, Dorfman VB, Loidl CF, Martínez A (2016) Cold Shock Proteins Are Expressed in the Retina Following Exposure to Low Temperatures. PloS one 11 (8).

39. Rey-Funes M, Larrayoz IM, Contartese DS, Soliño M, Sarotto A, Bustelo M, Bruno M, Dorfman VB, Loidl CF, Martínez A (2017) Hypothermia Prevents Retinal Damage Generated by Optic Nerve Trauma in the Rat. Scientific reports 7 (1).

40. Lewis ML, Reynolds JL, Cubano LA, Hatton JP, Lawless BD, Piepmeier EH (1998) Spaceflight Alters Microtubules and Increases Apoptosis in Human Lymphocytes (Jurkat). FASEB journal : official publication of the Federation of American Societies for Experimental Biology 12 (11).

41. Locatelli L, Cazzaniga A, De Palma C, Castiglioni S, Maier J (2020) Mitophagy contributes to endothelial adaptation to simulated microgravity. FASEB journal : official publication of the Federation of American Societies for Experimental Biology 34 (1).

42. Maier JA, Cialdai F, Monici M, Morbidelli L (2015) The impact of microgravity and hypergravity on endothelial cells. BioMed research international 2015.

43. Prasad B, Grimm D, Strauch SM, Erzinger GS, Corydon TJ, Lebert M, Magnusson NE, Infanger M, Richter P, Krüger M (2020) Influence of Microgravity on Apoptosis in Cells, Tissues, and Other Systems In Vivo and In Vitro. International journal of molecular sciences 21 (24).

44. Schatten H, Lewis ML, Chakrabarti A (2001) Spaceflight and clinorotation cause cytoskeleton and mitochondria changes and increases in apoptosis in cultured cells. Acta astronautica 49 (3-10).

45. Carlsson SI, Bertilaccio MT, Ballabio E, Maier J (2003) Endothelial stress by gravitational unloading: effects on cell growth and cytoskeletal organization. Biochimica et biophysica acta $1642(3)$.

46. Monici M, Fusi F, Paglierani M, Marziliano N, Cogoli A, Pratesi R, Bernabei PA (2006) Modeled gravitational unloading triggers differentiation and apoptosis in preosteoclastic cells. Journal of cellular biochemistry $98(1)$.

47. Kopp S, Slumstrup L, Corydon TJ, Sahana J, Aleshcheva G, Islam T, Magnusson NE, Wehland M, Bauer J, Infanger M, Grimm D (2016) Identifications of novel mechanisms in 
breast cancer cells involving duct-like multicellular spheroid formation after exposure to the Random Positioning Machine. Scientific reports 6.

48. Aleshcheva G, Sahana J, Ma X, Hauslage J, Hemmersbach R, Egli M, Infanger M, Bauer J, Grimm D (2013) Changes in morphology, gene expression and protein content in chondrocytes cultured on a random positioning machine. PloS one 8 (11).

49. Grimm D, Bauer J, Kossmehl P, Shakibaei M, Schöberger J, Pickenhahn H, SchulzeTanzil G, Vetter R, Eilles C, Paul M, Cogoli A (2002) Simulated microgravity alters differentiation and increases apoptosis in human follicular thyroid carcinoma cells. FASEB journal : official publication of the Federation of American Societies for Experimental Biology $16(6)$.

50. Hu J, Li Y, Hao Y, Zheng T, Gupta SK, Parada GA, Wu H, Lin S, Wang S, Zhao X, Goldman RD, Cai S, Guo M (2019) High stretchability, strength, and toughness of living cells enabled by hyperelastic vimentin intermediate filaments. Proceedings of the National Academy of Sciences of the United States of America 116 (35).

51. Bikle DD, Harris J, Halloran BP, Morey-Holton E (1994) Altered skeletal pattern of gene expression in response to spaceflight and hindlimb elevation. The American journal of physiology 267 (6 Pt 1).

52. Hammond TG, Lewis FC, Goodwin TJ, Linnehan RM, Wolf DA, Hire KP, Campbell WC, Benes E, O'Reilly KC, Globus RK, Kaysen JH (1999) Gene expression in space. Nature medicine 5 (4).

53. Li CF, Pan YK, Gao Y, Shi F, Wang YC, Sun XQ (2019) Autophagy protects HUVECs against ER stress-mediated apoptosis under simulated microgravity. Apoptosis : an international journal on programmed cell death 24 (9-10).

54. Jiang M, Wang H, Liu Z, Lin L, Wang L, Xie M, Li D, Zhang J, Zhang R (2020) Endoplasmic reticulum stress-dependent activation of iNOS/NO-NF-KB signaling and NLRP3 inflammasome contributes to endothelial inflammation and apoptosis associated with microgravity. FASEB journal : official publication of the Federation of American Societies for Experimental Biology 34 (8).

55. Zhang R, Jiang M, Zhang J, Qiu Y, Li D, Li S, Liu J, Liu C, Fang Z, Cao F (2020) Regulation of the cerebrovascular smooth muscle cell phenotype by mitochondrial oxidative injury and endoplasmic reticulum stress in simulated microgravity rats via the PERK-elF2a-ATF4-CHOP pathway. Biochimica et biophysica acta Molecular basis of disease 1866 (8).

56. Cazzaniga A, Locatelli L, Castiglioni S, Maier JAM (2019) The dynamic adaptation of primary human endothelial cells to simulated microgravity. FASEB journal : official publication of the Federation of American Societies for Experimental Biology 33 (5).

57. Gertz ML, Chin CR, Tomoiaga D, MacKay M, Chang C, Butler D, Afshinnekoo E, Bezdan D, Schmidt MA, Mozsary C, Melnick A, Garrett-Bakelman F, Crucian B, Lee SMC, Zwart SR, 
Smith SM, Meydan C, Mason CE (2020) Multi-omic, Single-Cell, and Biochemical Profiles of Astronauts Guide Pharmacological Strategies for Returning to Gravity. Cell reports 33 (10). 58. Han S, Chen J, Hua J, Hu X, Jian S, Zheng G, Wang J, Li H, Yang J, Hejtmancik JF, Qu J, Ma X, Hou L (2020) MITF protects against oxidative damage-induced retinal degeneration by regulating the NRF2 pathway in the retinal pigment epithelium. Redox biology 34.

59. Beheshti A, Ray S, Fogle H, Berrios D, Costes SV (2018) A microRNA signature and TGFbeta1 response were identified as the key master regulators for spaceflight response. PloS one 13 (7):e0199621.

60. Shi L, Tian H, Wang P, Li L, Zhang Z, Zhang J, Zhao Y (2020) Spaceflight and simulated microgravity suppresses macrophage development via altered RAS/ERK/NFKB and metabolic pathways. Cellular \& molecular immunology.

61. Dietz C, Infanger M, Romswinkel A, Strube F, Kraus A (2019) Apoptosis Induction and Alteration of Cell Adherence in Human Lung Cancer Cells under Simulated Microgravity. International journal of molecular sciences 20 (14).

62. Zhou M, Geathers JS, Grillo SL, Weber SR, Wang W, Zhao Y, Sundstrom JM (2020) Role of Epithelial-Mesenchymal Transition in Retinal Pigment Epithelium Dysfunction. Frontiers in cell and developmental biology 8 .

63. Ranieri D, Proietti S, Dinicola S, Masiello MG, Rosato B, Ricci G, Cucina A, Catizone A, Bizzarri M, Torrisi MR (2017) Simulated microgravity triggers epithelial mesenchymal transition in human keratinocytes. Scientific reports 7 (1).

64. Chen Z, Shao Y, Li X (2015) The roles of signaling pathways in epithelial-to-mesenchymal transition of PVR. Molecular vision 21.

65. Chen Y, Wu B, He JF, Chen J, Kang ZW, Liu D, Luo J, Fang K, Leng X, Tian H, Xu J, Jin C, Zhang J, Wang J, Zhang J, Ou Q, Lu L, Gao F, Xu GT (2021) Effectively Intervening Epithelial-Mesenchymal Transition of Retinal Pigment Epithelial Cells With a Combination of ROCK and TGF- $\beta$ Signaling Inhibitors. Investigative ophthalmology \& visual science 62 (4).

66. Du Y, Chen Q, Huang L, Wang S, Yin X, Zhou L, Ye Z, Ren X, Cai Y, Ding X, Ouyang H, Li X, Ju H (2018) VEGFR2 and VEGF-C Suppresses the Epithelial-Mesenchymal Transition Via YAP in Retinal Pigment Epithelial Cells. Current molecular medicine 18 (5).

67. Wilson SE (2021) TGF beta $-1,-2$ and -3 in the modulation of fibrosis in the cornea and other organs. Experimental eye research 207.

68. Moon S, Lee S, Caesar JA, Pruchenko S, Leask A, Knowles JA, Sinon J, Chaqour B (2020) A CTGF-YAP Regulatory Pathway Is Essential for Angiogenesis and Barriergenesis in the Retina. iScience 23 (6).

69. Lau LF (2016) Cell surface receptors for CCN proteins. Journal of cell communication and signaling 10 (2). 
70. Di Gesualdo F, Capaccioli S, Lulli M (2014) A pathophysiological view of the long noncoding RNA world. Oncotarget 5 (22):10976-10996.

71. Wang Y, Wang K, Zhang L, Tan Y, Hu Z, Dang L, Zhou H, Li G, Wang H, Zhang S, Shi F, Cao X, Zhang G (2020) Targeted overexpression of the long noncoding RNA ODSM can regulate osteoblast function in vitro and in vivo. Cell death \& disease 11 (2).

72. Fu H, Su F, Zhu J, Zheng X, Ge C (2020) Effect of simulated microgravity and ionizing radiation on expression profiles of miRNA, IncRNA, and mRNA in human lymphoblastoid cells. Life sciences in space research 24.

73. Thiel CS, Hauschild S, Huge A, Tauber S, Lauber BA, Polzer J, Paulsen K, Lier H, Engelmann F, Schmitz B, Schütte A, Layer LE, Ullrich O (2017) Dynamic gene expression response to altered gravity in human T cells. Scientific reports 7 (1).

74. Luo R, Li L, Hu YX, Xiao F (2021) LncRNA H19 inhibits high glucose-induced inflammatory responses of human retinal epithelial cells by targeting miR-19b to increase SIRT1 expression. The Kaohsiung journal of medical sciences 37 (2).

75. Thomas AA, Biswas S, Feng B, Chen S, Gonder J, Chakrabarti S (2019) IncRNA H19 prevents endothelial-mesenchymal transition in diabetic retinopathy. Diabetologia 62 (3).

76. Liang J, Liao J, Liu T, Wang Y, Wen J, Cai N, Huang Z, Xu W, Li G, Ding Z, Zhang B (2020) Comprehensive analysis of TGF- $\beta$-induced mRNAs and ncRNAs in hepatocellular carcinoma. Aging 12 (19).

77. Zhang LC, Wei ZB, Tang SF (2020) Knockdown of the Long Noncoding RNA LUCAT1 Inhibits High-Glucose-Induced Epithelial-Mesenchymal Transition through the miR-199a-5pZEB1 Axis in Human Renal Tubular Epithelial Cells. BioMed research international 2020.

78. Malkani S, Chin CR, Cekanaviciute E, Mortreux M, Okinula H, Tarbier M, Schreurs AS, Shirazi-Fard Y, Tahimic CGT, Rodriguez DN, Sexton BS, Butler D, Verma A, Bezdan D, Durmaz C, MacKay M, Melnick A, Meydan C, Li S, Garrett-Bakelman F, Fromm B, Afshinnekoo E, Langhorst BW, Dimalanta ET, Cheng-Campbell M, Blaber E, Schisler JC, Vanderburg C, Friedländer MR, McDonald JT, Costes SV, Rutkove S, Grabham P, Mason CE, Beheshti A (2020) Circulating miRNA Spaceflight Signature Reveals Targets for Countermeasure Development. Cell reports 33 (10).

79. Kasiviswanathan D, Chinnasamy Perumal R, Bhuvaneswari S, Kumar P, Sundaresan L, Philip M, Puthenpurackal Krishnankutty S, Chatterjee S (2020) Interactome of miRNAs and transcriptome of human umbilical cord endothelial cells exposed to short-term simulated microgravity. NPJ microgravity 6.

80. Qin W, Liu L, Wang Y, Wang Z, Yang A, Wang T (2019) Mir-494 inhibits osteoblast differentiation by regulating BMP signaling in simulated microgravity. Endocrine 65 (2). 
81. Gong L, Wu X, Li X, Ni X, Gu W, Wang X, Ji H, Hu L, Zhu L (2020) S1PR3 deficiency alleviates radiation-induced pulmonary fibrosis through the regulation of epithelialmesenchymal transition by targeting miR-495-3p. Journal of cellular physiology 235 (3).

82. Ma Y, Duan J, Hao X (2020) Down-regulated HDAC3 elevates microRNA-495-3p to restrain epithelial-mesenchymal transition and oncogenicity of melanoma cells via reducing TRAF5. Journal of cellular and molecular medicine 24 (22).

83. Jiang H, Huang G, Zhao N, Zhang T, Jiang M, He Y, Zhou X, Jiang X (2018) Long noncoding RNA TPT1-AS1 promotes cell growth and metastasis in cervical cancer via acting AS a sponge for miR-324-5p. Journal of experimental \& clinical cancer research : CR 37 (1).

84. Zhang Q, Liu H, Zhang J, Shan L, Yibureyimu B, Nurlan A, Aerxiding P, Luo Q (2020) MiR142-5p Suppresses Lung Cancer Cell Metastasis by Targeting Yin Yang 1 to Regulate Epithelial-Mesenchymal Transition. Cellular reprogramming 22 (6).

85. Zhang PF, Wang F, Wu J, Wu Y, Huang W, Liu D, Huang XY, Zhang XM, Ke AW (2019) LncRNA SNHG3 induces EMT and sorafenib resistance by modulating the miR-128/CD151 pathway in hepatocellular carcinoma. Journal of cellular physiology 234 (3).

86. Zhang X, Liu L, Deng X, Li D, Cai H, Ma Y, Jia C, Wu B, Fan Y, Lv Z (2019) MicroRNA 483-3p targets Pard3 to potentiate TGF- $\beta 1$-induced cell migration, invasion, and epithelialmesenchymal transition in anaplastic thyroid cancer cells. Oncogene 38 (5).

87. Qu J, Kaufman Y, Washington I (2009) Coenzyme Q10 in the human retina. Investigative ophthalmology \& visual science 50 (4).

88. Li WY, Zhou HZ, Chen Y, Cai XF, Tang H, Ren JH, Wai Wong VK, Kwan Law BY, Cheng ST, Yu HB, Cai HY, Chen WX, Tang N, Zhang WL, Tao NN, Yang QX, Ren F, He L, Jiang H, Huang AL, Chen J (2019) NAD(P)H: Quinone oxidoreductase 1 overexpression in hepatocellular carcinoma potentiates apoptosis evasion through regulating stabilization of $X$ linked inhibitor of apoptosis protein. Cancer Lett 451:156-167.

89. Wang T, Chen H, Lv K, Ji G, Liang F, Zhang Y, Wang Y, Liu X, Cao H, Kan G, Xiong J, Li $Y, Q u L$ (2017) Activation of HIF-1a and its downstream targets in rat hippocampus after longterm simulated microgravity exposure. Biochemical and biophysical research communications 485 (3).

90. Vogel J, Thiel CS, Tauber S, Stockmann C, Gassmann M, Ullrich O (2019) Expression of Hypoxia-Inducible Factor $1 \alpha$ (HIF-1 $\alpha$ ) and Genes of Related Pathways in Altered Gravity. International journal of molecular sciences 20 (2).

91. Dixon SJ, Lemberg KM, Lamprecht MR, Skouta R, Zaitsev EM, Gleason CE, Patel DN, Bauer AJ, Cantley AM, Yang WS, Morrison B, Stockwell BR (2012) Ferroptosis: an irondependent form of nonapoptotic cell death. Cell 149 (5). 
92. Alvarez SW, Sviderskiy VO, Terzi EM, Papagiannakopoulos T, Moreira AL, Adams S, Sabatini DM, Birsoy K, Possemato R (2017) NFS1 undergoes positive selection in lung tumours and protects cells from ferroptosis. Nature 551 (7682).

93. Dixon SJ, Patel DN, Welsch M, Skouta R, Lee ED, Hayano M, Thomas AG, Gleason CE, Tatonetti NP, Slusher BS, Stockwell BR (2014) Pharmacological inhibition of cystineglutamate exchange induces endoplasmic reticulum stress and ferroptosis. eLife 3.

94. Santoro M (2020) The Antioxidant Role of Non-mitochondrial CoQ10: Mystery Solved! Cell metabolism 31 (1). 


\section{Figures}

Figure 1

a

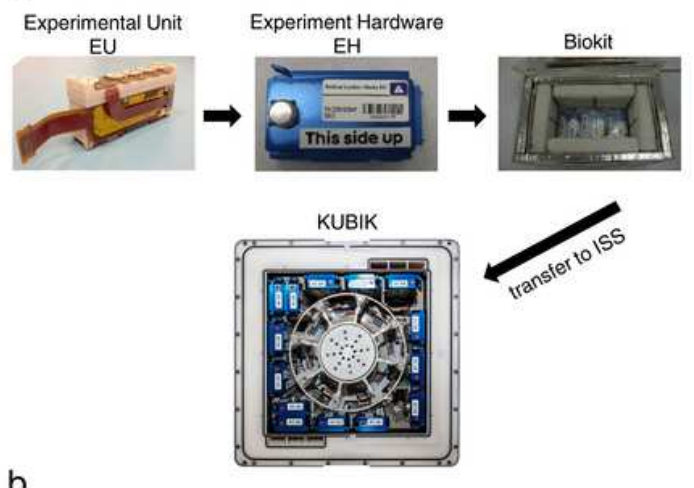

b

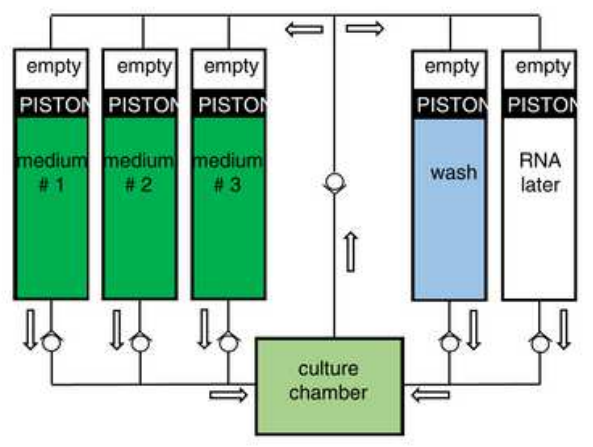

C

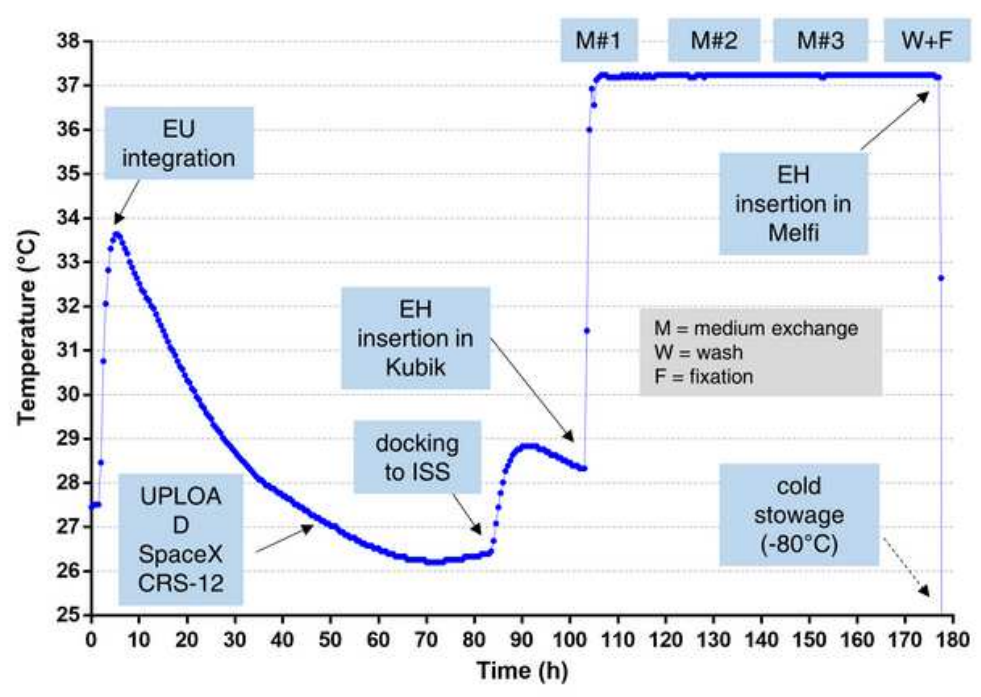

Figure 1

A) The hardware of CORM experiment: the Experimental Units (EU) were integrated into Experimental Container to compose the Experiment Hardware (EH), which were placed inside the Biokit transportation container that was launched to the ISS. Once onboard ISS, the EH were inserted into the ESA KUBIK incubator in the ISS Columbus module. B) Schematic fluidic of the EU. C) iButton data logger-derived temperature profile of the CORM experiment. 
Figure 2

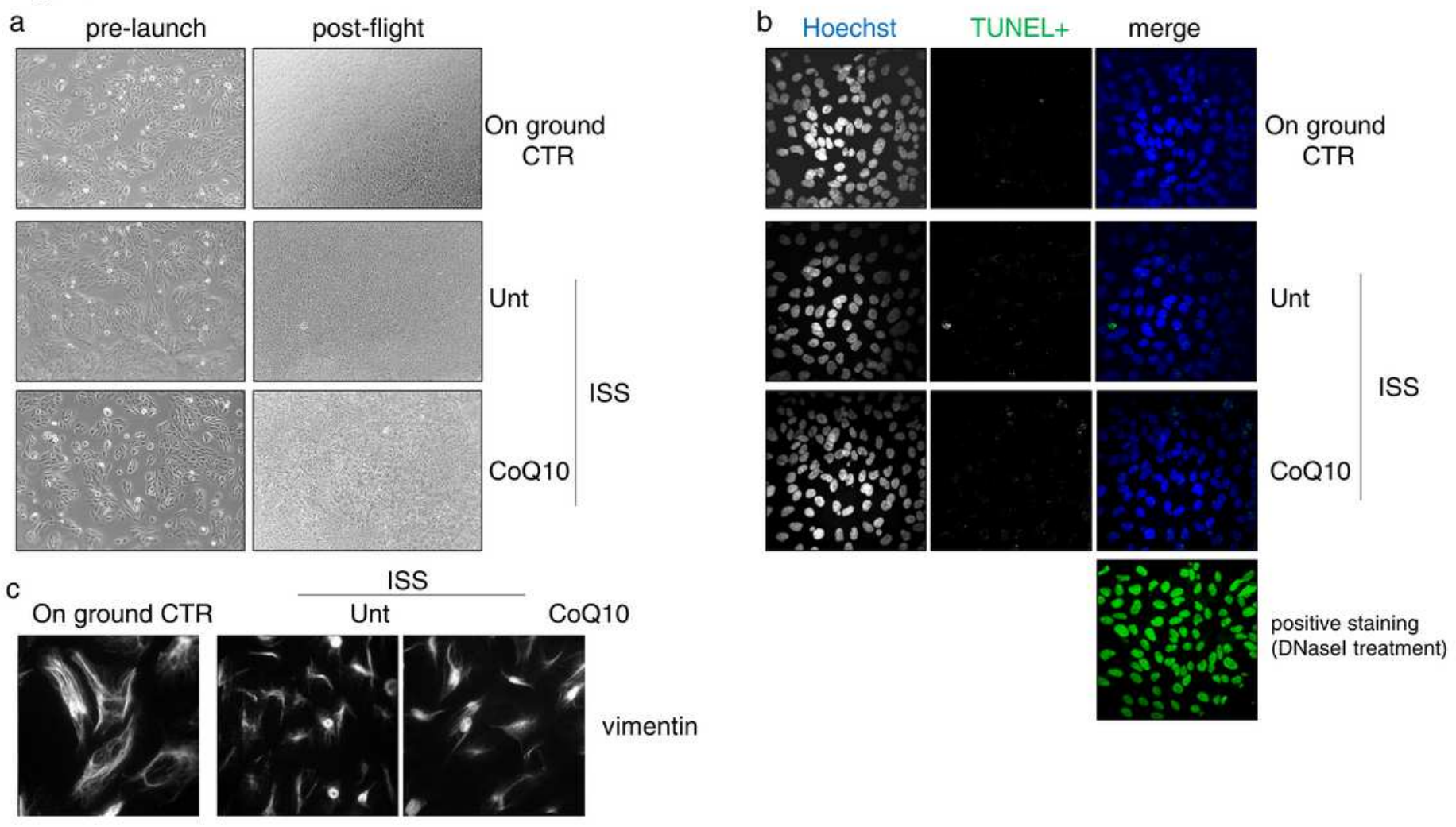

Figure 2

A) Representative photomicrographs obtained with phase contrast microscopy in ARPE-19 cell pre-launch and post-flight. B) Evaluation of apoptosis in ARPE-19 cells by TUNEL fluorescent staining. Image size: 250x250 um. C) Evaluation of vimentin cellular localization in ARPE-19 cells by immunofluorescence. Image size: 100x100 um. 
Figure 3

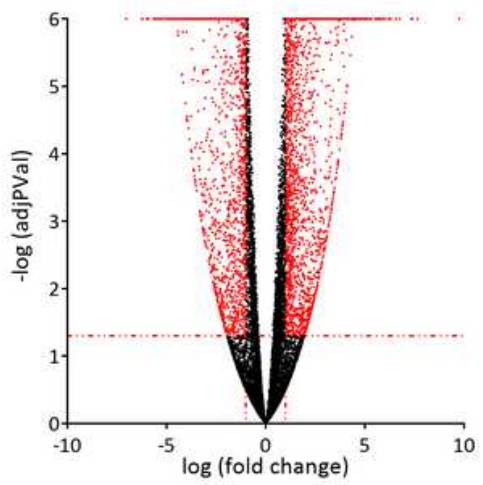

b

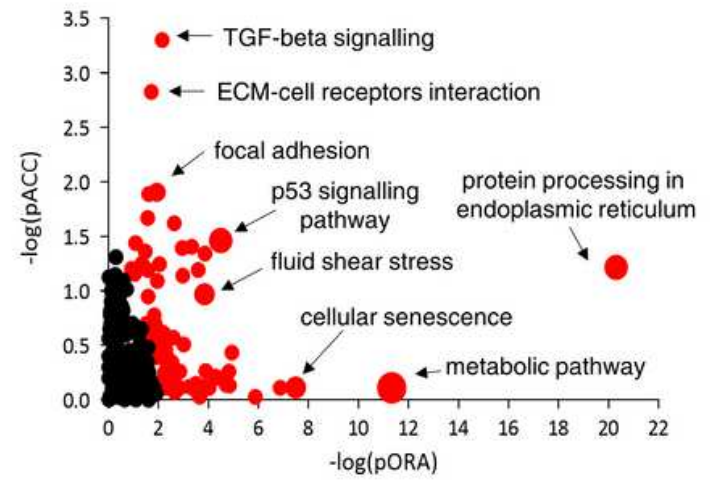

C

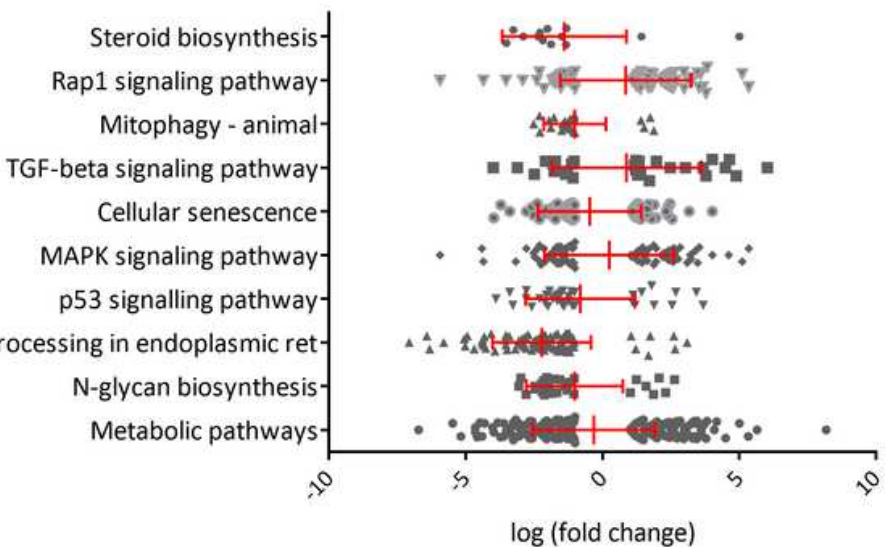

\section{Figure 3}

A) The 5555 significantly DE genes (shown in red) are represented in the Volcano plot in terms of their measured expression change (x-axis) and the significance of the change (y-axis). The significance is represented in terms of the negative log (base 10) of the p-value, so that genes that are more significant are plotted higher on the y-axis. The dotted lines represent the thresholds used to select the DE genes: 1 for expression change and 0.05 for significance. B) Pathways perturbation vs over-representation: the pathways are plotted in terms of the two types of evidence computed by iPathwayGuide: overrepresentation is on the $x$-axis (pORA) and the total pathway accumulation is on the $y$-axis (pAcc). Each pathway is represented by a single dot, with significant pathways shown in red, non-significant in black, and the size of each dot is proportional to the size of the pathway it represents. Both p-values are shown in terms of their negative log (base 10) values. C) Differentially expressed pathway genes of the most significant impacted pathways. Mean value and SD are graphed in red. 
Figure 4

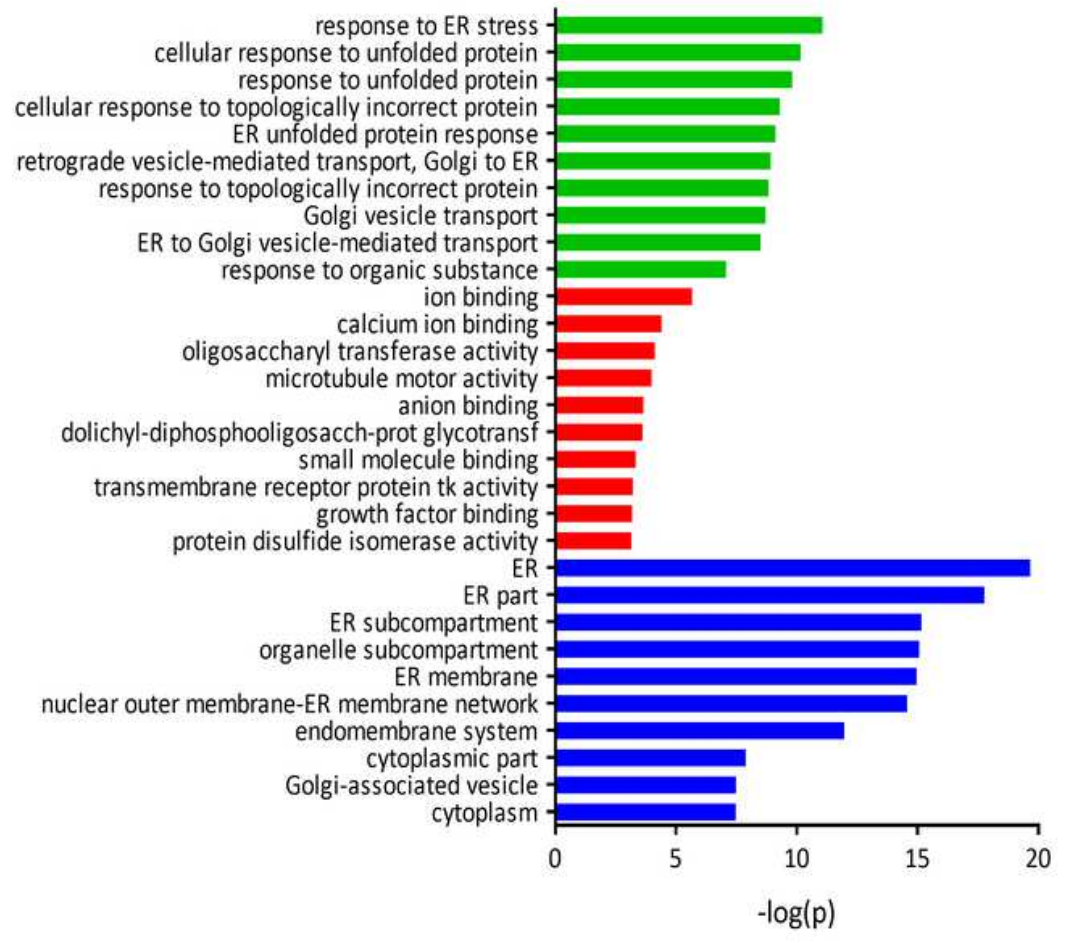

\section{Figure 4}

GO analysis of DE genes; top ten GO terms in biological processes (green), molecular functions (red) and cellular components (blue) category are listed.

Figure 5

a

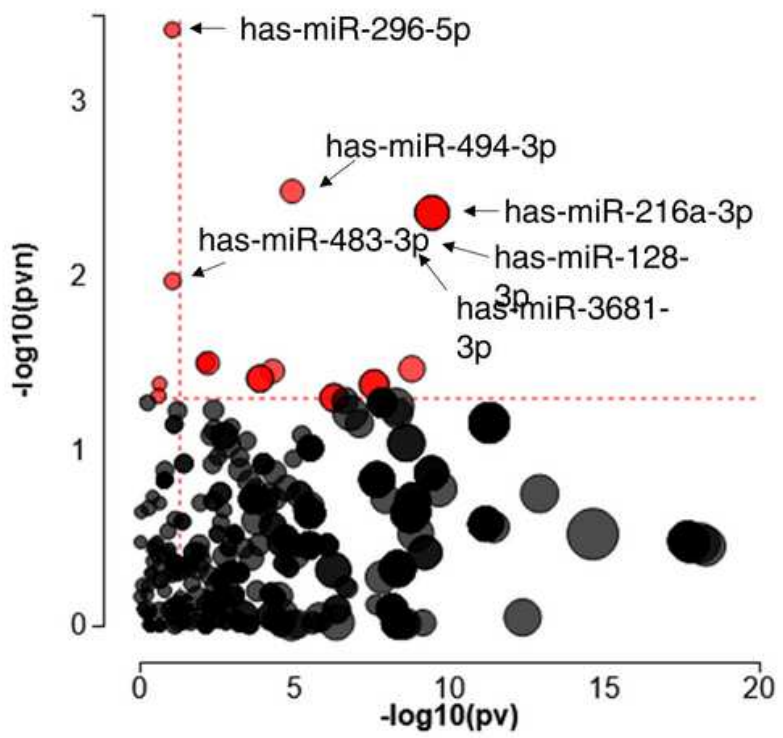

b

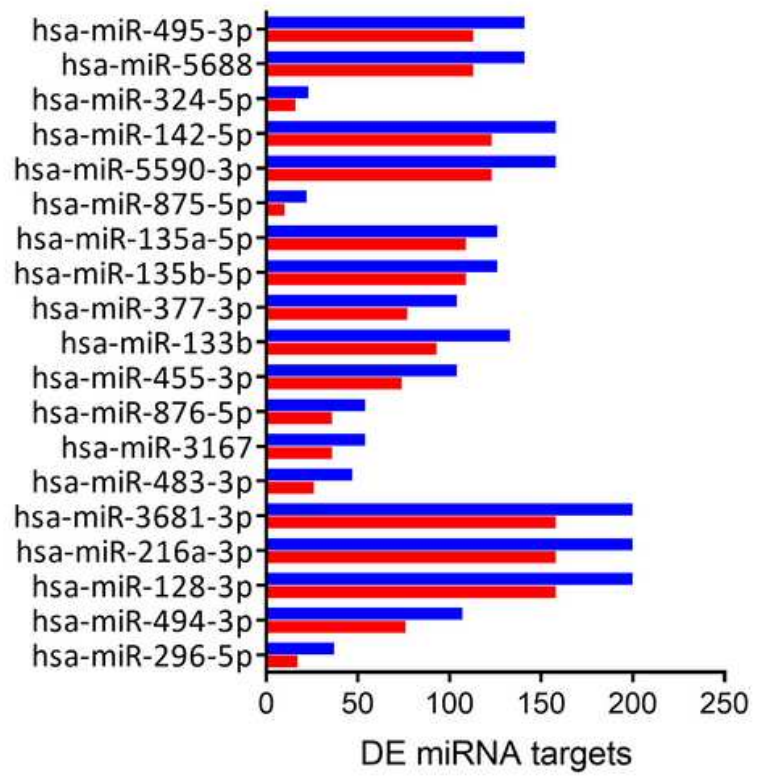




\section{Figure 5}

A) Significant differences in specific miRNA expression plotted in term of the two types of evidence computed by iPathwayGuide: the $x$-axis $(-\log 10(p v))$ representing the $p$-value based on the total number of DE target genes versus the total number of target genes and the $y$-axis $(-\log 10(p v n))$ is the $p$-value based on the number of downwardly expressed DE targets versus the total number of DE miRNA targets. Each miRNA is represented by a single dot, with significant miRNAs shown in red, non-significant in black, and the size of each dot is proportional to the size of the number of target genes for that miRNA relative to other ones. B) List of the 19 miRNAs which reached significant expression values, depicting the number of DE target genes that are under-expressed (in blue) or overexpressed (in red). 


\section{Figure 6}
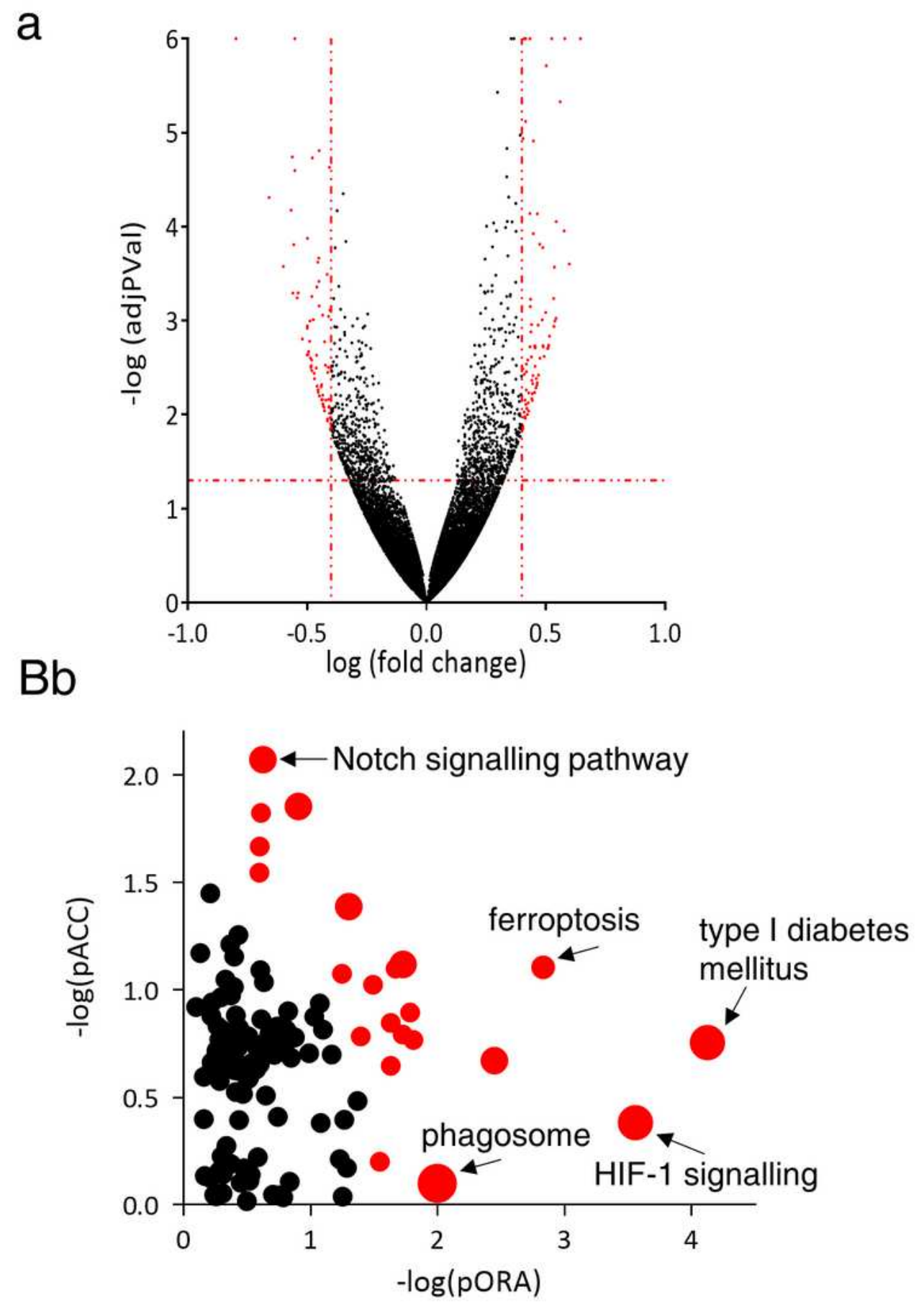

Figure 6

A) All 153 significantly DE genes (showed in red) are represented in the Volcano plot in terms of their measured expression change ( $x$-axis) and the significance of the change (y-axis), as for Figure 3A. The dotted lines represent the thresholds used to select the DE genes: 0.4 for expression change and 0.05 for significance. B) Pathways perturbation vs over-representation. Each pathway is represented by a single dot, with significant pathways shown in red, non-significant in black, and the size of each dot is 
proportional to the size of the pathway it represents. Both $p$-values are shown in terms of their negative $\log ($ base 10) values.

\section{Figure 7}

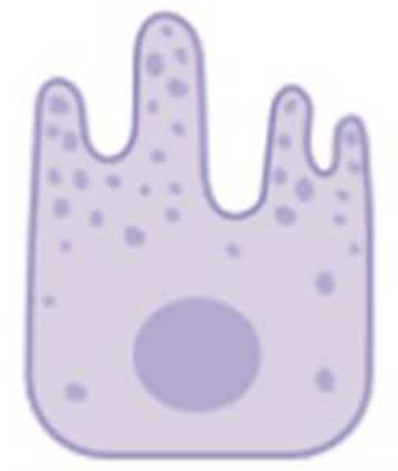

retinal pigment epithelial cells vimentin network dysorganization

$\checkmark$ deregulation of:

- protein processing in ER

- mitophagy

- TGF-beta signaling

- Hyppo signaling

- p53 signaling

- senescence pathway

$\checkmark$ alteration of IncRNA expression
CoQ10
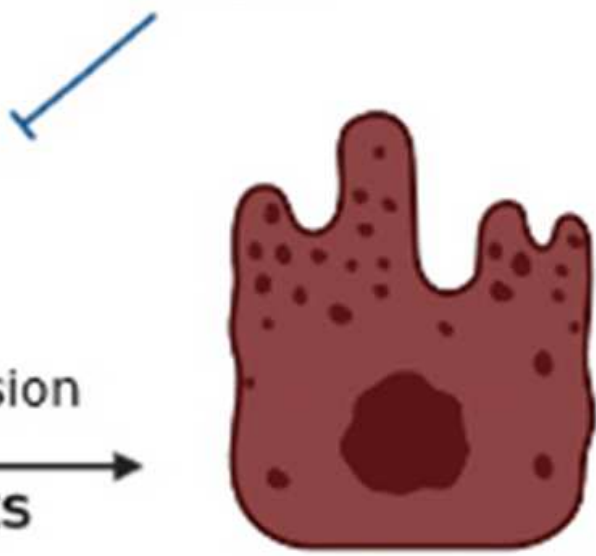

cellular dysfunction

Figure 7

Space flight effects on ARPE-19 cells.

\section{Supplementary Files}

This is a list of supplementary files associated with this preprint. Click to download.

- SupplementaryS1.xlsx

- SupplementaryS2.xIsx

- SupplementaryS3.pptx

- SupplementaryS4.xIsx

- SupplementaryS5.xIsx

- SupplementaryS6.xlsx

- SupplementaryS7.xlsx

- SupplementaryS8.xIsx 